\title{
Pratique de l'apprentissage interculturel
}

L'exploration de concepts par de futurs enseignants norvégiens

The practice of intercultural learning. The exploration of concepts by prospective teachers in Norway

Práctica del aprendizaje intercultural. La exploración de conceptos por futuros profesores noruegos

\section{Lise Kvande et Claudia Lenz}

Traducteur : Robert Elbaz

\section{CpenEdition}

\section{Journals}

Édition électronique

URL : http://journals.openedition.org/ries/3495

DOI : 10.4000/ries.3495

ISSN : 2261-4265

Éditeur

Centre international d'études pédagogiques

Édition imprimée

Date de publication : 1 septembre 2013

Pagination : 111-121

ISBN : 978-2-85420-600-5

ISSN : 1254-4590

Référence électronique

Lise Kvande et Claudia Lenz, «Pratique de l'apprentissage interculturel », Revue internationale

d'éducation de Sèvres [En ligne], 63 | septembre 2013, mis en ligne le 01 septembre 2015, consulté le 01 mai 2019. URL : http://journals.openedition.org/ries/3495 ; DOI : 10.4000/ries.3495

(c) Tous droits réservés 


\title{
Pratique de l'apprentissage interculturel
}

\author{
L'exploration de concepts \\ par de futurs enseignants norvégiens*
}

\author{
Lise Kvande \\ Claudia Lenz
}

\section{SE PRÉPARER À UNE diVERSITÉ CULTURELLE ACCRUE DANS LES ÉCOLES NORVÉGIENNES}

En Norvège, comme partout ailleurs, le curriculum varie selon les changements sociétaux. Au cours des dernières décennies, la Norvège a été confrontée à une immigration croissante. Les classes, à l'origine essentiellement homogènes culturellement, se sont diversifiées. La réforme du curriculum de 2006 a intégré ces changements, mettant davantage l'accent sur la compréhension et la tolérance interculturelles ${ }^{1}$. On s'appuie sur ces qualités dans les principes éducatifs généraux ainsi que dans les objectifs spécifiques de compétences à acquérir dans plusieurs matières. Cependant, la mise en œuvre dans les classes n'a pas encore fait l'objet d'études véritables. Les didacticiens de chaque matière commencent tout juste à reformuler la façon dont les groupes d'élèves multiculturels mettent à l'épreuve les contenus d'enseignement, afin de favoriser le processus d'intégration des nouveaux groupes culturels. Jusqu'à présent, la plus grande partie de la recherche pédagogique et des manuels destinés à la " nouvelle » Norvège ne traitaient que de perspectives générales portant sur l'intégration, la tolérance, le défi linguistique et les méthodes destinées à faire coopérer des élèves provenant de contextes culturels distincts. Le défi suivant est d'approfondir la façon dont une classe multiculturelle pourrait et devrait fonctionner, grâce à un contenu reformulé matière par matière. Plus important encore, ce défi s'adresse aux enseignants en sciences sociales tout autant qu'à ceux des disciplines littéraires (langues et histoire). Jusqu'à présent, ces questions demeurent tout à la fois un défi et un problème puisqu'il n'existe qu'un nombre limité de publications scientifiques et/ou fondées sur la pratique, et de recommandations politiques claires. Certes, il existe une véritable volonté et un consensus politique pour créer une société bien intégrée tout en étant culturellement

\footnotetext{
* Article traduit par Robert Elbaz.

1. Central : National Curriculum for Knowledge Promotion in Primary and Secondary Education and Training/ Quality Framework, p. 3 [Curriculum national pour la promotion des connaissances dans l'enseignement et la formation primaire et secondaire / Cadre général relatif à la qualité $(N d T)]$.
} 
diversifiée grâce au système éducatif, mais les moyens d'y parvenir demeurent flous. Ainsi, les enseignants et les formateurs doivent déterminer par eux-mêmes ce que signifie l'apprentissage interculturel, en théorie comme en pratique.

\section{LES COMPÉTENCES NUMÉRIQUES}

La partie générale du curriculum norvégien précise que cinq compétences fondamentales doivent être développées pour l'ensemble des matières. Il s'agit de la lecture, de l'expression orale et écrite, de la numéracie et des compétences numériques. Ces compétences numériques sont principalement définies comme étant la capacité à utiliser des sources Internet de façon critique et à se comporter en ligne de façon éthique et appropriée. La possibilité d'utiliser Internet pour communiquer et pour discuter avec des camarades à l'extérieur de la classe est à peine mentionnée. En conséquence, on suppose que les compétences numériques sont une affaire individuelle face à la publication d'informations. Le potentiel qu'a Internet de favoriser le dialogue interculturel est certes mentionné mais il ne fait l'objet d'aucun approfondissement. Un fossé semble exister entre l'utilisation que les élèves font d'Internet et les visées pédagogiques des compétences numériques : les élèves participent activement à des réseaux sociaux tels que Facebook et Twitter, par l'intermédiaire desquels ils nouent et maintiennent des contacts avec les jeunes du monde entier. Cependant, cette manière informelle d'établir une communication interculturelle n'est pas considérée comme une ressource potentielle d'enseignement interculturel dans les écoles. Ainsi, les relations sociales créées et maintenues par l'intermédiaire des réseaux sociaux sont considérées comme "privées » et "sociales " plutôt que comme une occasion d'enseignement et de développement formels par le biais d'un dialogue interculturel.

Cette situation n'est pas spécifiquement norvégienne. Partout ailleurs, les enseignants et les formateurs sont confrontés au même fossé séparant les possibilités d'Internet et le cadre fixé pour l'enseignement de chaque matière, dans un temps limité et avec la nécessité d'atteindre des objectifs spécifiques en termes de compétences. Compte tenu de la richesse du potentiel d'Internet, c'est regrettable. Cela permet aussi d'évaluer la façon dont les établissements parviennent à prendre en compte la vie contemporaine et les défis auxquels les élèves sont confrontés. Sans aller jusqu'à dire que tout enseignement doit être «cool» et «populaire », l'absence de cohérence entre l'enseignement et les formes de communication propres aux élèves signifie de fait que les apprentissages et les compétences numériques n'ont plus aucun rapport avec la vie et l'expérience personnelles des élèves.

La communication et la coopération avec les élèves d'autres écoles et d'autres pays sont également mentionnées dans la description de ces compétences fondamentales. Cependant, on ne dispose que de très peu d'indications sur la façon dont celles-ci doivent être développées dans les écoles. On peut 
raisonnablement supposer que cette part de la compétence numérique n'est pas approfondie dans les classes, l'une des raisons étant sans doute la compétence insuffisante des enseignants pour établir une telle communication, l'autre étant la lourdeur de l'investissement en temps et en travail que la recherche de partenaires extérieurs à l'établissement exige, lorsque de tels réseaux n'existent pas déjà. On peut donc penser que ce n'est pas le manque de bonne volonté mais plutôt l'absence de conditions propices qui empêche actuellement de répondre aux exigences du curriculum.

\section{Le Projet de glossaire INTERCULTUREL}

"Que signifie ce mot?» est une question fréquente dans les classes norvégiennes. La compréhension qu'ont les élèves des manuels scolaires et de l'enseignement dispensé est étroitement tributaire de la compréhension des mots et des concepts utilisés. Les curricula norvégiens mettent l'accent sur l'apprentissage et la compréhension de mots et de concepts spécifiques, qui sont les principales compétences à acquérir de 10 à 19 ans. En accord avec la taxonomie de Bloom (1956), ces compétences vont de «l'utilisation » à la " définition » ou à "l'élaboration » de mots et de concepts. Les connaissances et les compétences nécessaires à la compréhension conceptuelle aident les élèves à comprendre les contenus des enseignements et leur permettent de débattre de façon critique de concepts à l'extrême pointe des problématiques politiques et du développement socioculturel contemporains. On peut supposer que les pratiques de classe permettent rarement aux élèves de se demander ce que les concepts clés signifient et comment ils agissent, dans la communication quotidienne comme dans le débat public. La réponse à la question "Que signifie ce mot ? » est trop souvent une définition fermée, correspondant à une demande de réponses toutes faites et de références en vue des examens futurs.

Le Projet de glossaire interculturel (ou PGI) ${ }^{2}$ vise à développer de nouveaux outils pour la compréhension, l'exploration et la discussion de concepts clés. En ce qui concerne la formation des maîtres, le PGI cherche à donner aux futurs enseignants des méthodes pour explorer et pour débattre de concepts clés avec leurs élèves. Les concepts clés sont des termes qui, tout en étant difficiles à définir, sont essentiels pour la compréhension et le questionnement de la société contemporaine, tels que "démocratie ", "égalité des droits ", " citoyenneté ", " droits de l'homme », " ethnicité », " valeurs universelles », "État-providence », "pouvoir politique» ou «mondialisation». Le terme "démocratie», par exemple, sera en grande partie compris de façon différente en France, en Égypte et en Chine, mais aussi entre citoyens de ces mêmes États.

2. The Intercultural Glossary project : www.interculturalglossary.net et, pour plus d'informations : www.bpb.de/ veranstaltungen/netzwerke/nece/154768/intercultural-glossary-project-igp. À partir de l'automne 2013, le projet s'intitulera "Concept Learning for Empowerment through Analysis and Reflection/CLEAR" et disposera d'un nouveau site Internet : www.clear-project.net 
Les concepts clés sont importants dans le débat public, et leur maîtrise fait partie de l'apprentissage de la citoyenneté. En même temps, ils sont souvent obscurs et font référence à des sens différents et parfois contradictoires qui dépendent de leurs contextes géographique, social et culturel, ainsi que de l'âge, des opinions politiques et des convictions personnelles de la personne qui les utilise. Enfin, et en raison de leur large éventail de sens et d'utilisations, les concepts clés sont toujours contestés. En d'autres termes, ils sont le lieu de désaccords, et parfois même de conflits, sociaux, politiques et culturels. Au lieu de présupposer l'existence de définitions universelles, nous partirons de l'hypothèse que la variété des façons de "faire sens " à partir de ces termes et de ces concepts est une ressource importante, dans le cadre de processus d'apprentissage cherchant à favoriser une pensée critique, une réflexion et des compétences interculturelles.

\section{VISIONS ET MÉTHODOLOGIE}

La vision de ce projet de glossaire interculturel est fondée sur les principes suivants :

- l'élaboration commune de la connaissance: encourager les apprenants à prendre au sérieux leurs propres perception et compréhension de ces concepts ; les autoriser à prendre part à l'examen critique des compréhensions préexistantes; générer de nouvelles connaissances;

- le déplacement de la priorité, de la vérité au sens : au lieu de chercher à se "débarrasser " des interprétations erronées en fournissant des réponses fermes et définitives, l'accent est mis sur la compréhension de différentes sources et de différents systèmes de sens; les processus d'apprentissage visent la clarification, non pas dans le sens de définitions consensuelles mais dans celui de la prise de conscience du caractère contextuel et de l'historicité des concepts ;

- l'encouragement au jugement critique: même si le projet repose sur une conception «faible» de la vérité, il n'implique pas pour autant un relativisme absolu. Par ses méthodes de travail, il cherche à mettre à l'épreuve la capacité qu'ont les apprenants à comparer et à juger de façon critique différentes assertions, leur validité, et les conséquences qu'ont certaines notions et concepts sur la réalité sociale et le pouvoir politique, ce qui n'est pas le moins difficile.

Le PGI, qui permet d'articuler interculturel et alphabétisation numérique, comprend trois éléments :

- la mise au point d'une plate-forme interactive en ligne, facilitant les processus d'apprentissage interactifs et collaboratifs ;

- la mise au point d'une méthodologie d'apprentissage conceptuelle réflexive (en ligne et hors ligne);

- l'organisation d'activités d'apprentissage en commun incluant des étudiants de différents pays lors des tests, des évaluations et des développements ultérieurs des points précédents. 
La méthodologie du PGI est composée de différentes étapes explorant différents sens attribués au fil du temps à un concept donné, dans différentes cultures. Le point de départ invariable est de demander aux apprenants comment ils comprennent le concept et de les inviter à réfléchir à ce qui a influencé leur propre compréhension, ainsi que celle des autres, lors du processus d'apprentissage. Les étapes suivantes de l'exploration ont été fortement structurées par l'histoire conceptuelle et par son fondateur, Reinhardt Koselleck. L'histoire conceptuelle s'intéresse à la façon dont le sens d'un concept se construit lors de circonstances politiques et socio-économiques données (Koselleck, $1972 ; 2002$ ). Ces concepts reflètent la manière dont une société "fait sens " à partir de la réalité. Ils reflètent cette réalité et participent à sa construction. Mais, puisque la façon dont le sens se construit est directement liée à l'action et aux intermédiaires qu'elle met en œuvre, les concepts sont le lieu d'une lutte permanente pour leur définition. L'analyse des concepts comme véhicules de sens est une façon de parvenir à l'action politique en permettant au sujet de se relier de façon active aux négociations de sens qui font partie intégrante de la culture politique d'une société, et d'y participer. Ce point est encore plus important dans les sociétés pluriculturelles, dans lesquelles les acteurs participant au débat politique et la prise de décisions doivent pouvoir "lire " la manière dont les autres décisionnaires et agents perçoivent les problèmes qui sont débattus.

Les principes de l'analyse synchronique et diachronique, établis par l'histoire conceptuelle, ont été rendus opérationnels sous la forme d'étapes dans la méthodologie d'apprentissage du PGI. Les apprenants explorent différents sens et définitions d'un concept donné et les placent dans une matrice, visualisant ainsi leur développement dans le temps et dans des contextes différents. Lorsque ceci est conduit de manière comparative, en comprenant des sources provenant de pays différents, le processus d'apprentissage peut déboucher à la fois sur une conscience accrue de soi-même et de la relativité de ses propres perceptions, et d'une compréhension aiguisée des différences et des ressemblances culturelles, ainsi que des implications politiques de ces perceptions et de cette compréhension.

\section{ÉTUdE DE CAS : UN PROCESSUS BINATIONAL D'APPRENTISSAGE ENTRE FUTURS ENSEIGNANTS}

Dans sa partie norvégienne, le projet PGI est conduit dans le cadre du master en didactique des sciences sociales de l'Université de Trondheim (NTNU). Le master qualifie les étudiants pour enseigner les sciences sociales et l'histoire dans les lycées. Le développement des compétences démocratiques, civiques et interculturelles est une perspective transversale de l'ensemble du programme du master. 
Au cours du troisième trimestre 2012, les étudiants ont exploré différents sens des concepts de citoyenneté, de liberté et de liberté de parole en utilisant la plate-forme du PGI. Ce même groupe d'étudiants a continué le travail du PGI lors d'un cours intitulé "Identité et politique ", qui s'est déroulé au premier trimestre 2013. Mais cette fois, ils ont interagi avec un groupe d'étudiants de l'Université du Luxembourg.

Il convient d'apporter quelques précisions sur la composition des deux groupes d'étudiants, puisque celle-ci a eu un impact significatif sur l'ensemble des interactions et sur le sens de l'expérience, dans le cadre de la préparation des futurs enseignants norvégiens à devenir des facilitateurs de l'apprentissage interculturel. Le groupe d'étudiants norvégiens peut être décrit comme plutôt homogène, à la fois en termes d'ethnicité et de contexte socioculturel. Durant l'exploration des concepts liés à la démocratie et à la citoyenneté, ils ont pris conscience de partager une vision très proche de ces concepts, mettant ainsi l'accent sur un processus de socialisation politiquement identique dans leurs familles, dans le système scolaire norvégien, etc.

Par contraste, le cours luxembourgeois faisait partie d'un programme de master multilingue (acceptant le français, l'allemand, le luxembourgeois et l'anglais comme langues de travail) et les étudiants qui y assistaient venaient de plus de dix pays différents, européens et asiatiques. Dans ce groupe, les premières réflexions sur ce concept ont presque naturellement consisté en une exploration des différents sens que ces concepts ont dans les différentes langues maternelles des étudiants présents.

Chaque groupe a eu une session initiale hors ligne indépendante, au cours de laquelle il a réfléchi sur ses propres hypothèses liées aux termes « communauté », "peuple», «normalité » et «minorité ». Après avoir téléchargé le résultat de leur travail sur la plate-forme en ligne, des étudiants du Luxembourg et de la NTNU ont échangé leurs opinions pendant douze jours sur la plateforme du PGI. Finalement, ils se sont rencontrés au cours d'un atelier d'un jour et demi au Luxembourg, tout en continuant à travailler sur ces concepts.

\section{Préparation hors ligne}

L'un des principes méthodologiques du PGI est d'utiliser la façon dont l'apprenant comprend le concept exploré comme point de départ du processus d'apprentissage. On considère ainsi les apprenants comme des agents contribuant de façon pertinente au processus d'apprentissage et, en même temps, on prépare le terrain à une réflexion sur soi concernant les différentes manières dont le contexte socioculturel donne forme à l'interprétation et à la compréhension de ces concepts. On a demandé aux étudiants de cartographier les concepts qu'ils avaient choisis. Par exemple, l'un des étudiants qui avaient choisi le concept «peuple» faisait une différence entre les notions ethnique et "populiste» du concept, l'une associée à la nation, l'autre aux idées de l'homme de la rue, mais également connotée de façon négative. 
On a demandé aux étudiants, travaillant en binôme, de mettre au point une première entrée, à poster sur la plate-forme en ligne. Les étudiants norvégiens n'ont pas été confrontés à des surprises particulières lors des premières étapes de ce processus. Cependant, les découvertes surprenantes n'ont pas manqué de se produire lors des échanges en ligne avec les étudiants luxembourgeois. Ceux-ci, fonctionnant en groupe multilingue, avaient exploré un certain nombre de différences dans leur approche, associées notamment à leurs différentes langues maternelles, ce qui a influencé et enrichi l'étape suivante du processus.

\section{Échanges en ligne}

Durant douze jours, les étudiants de Trondheim et du Luxembourg ont travaillé ensemble à la mise au point de la plate-forme en ligne. Cette coopération était structurée par trois tâches :

- télécharger sur le site les premières idées et les définitions de travail développées lors de la préparation;

- commenter ces données ;

- collecter des exemples d'interprétations et de définitions contemporaines et historiques du concept en cours d'exploration.

Ce faisant, il s'agissait d'assurer une progression dans le processus d'apprentissage, depuis la réflexion individuelle jusqu'à l'inclusion et l'exploration de différentes sources, en passant par l'échange de perspectives.

Les contributions mises sur le site ont immédiatement déclenché un échange de commentaires, au cours duquel le positionnement des participants (leur contexte personnel et leur langue maternelle) a joué un rôle important.

Un exemple intéressant de la dynamique de l'apprentissage interculturel qui a émergé, lors de l'échange en ligne, est la discussion portant sur le mot " minorité ». Les premiers posts téléchargés sur la plate-forme ont rapidement conduit à un échange de vues animé, au cours duquel la question des aspects quantitatif et qualitatif du terme a été débattue : "minorité » signifie-t-il (toujours) être la partie "mineure» en termes de nombre ou (toujours) " mineur» en termes de pouvoir ? Cela a conduit à une brève séquence d'échanges entre étudiants norvégiens, rejoints ensuite par un Bulgare et un étudiant portugais du Luxembourg :

G. : D’habitude, lorsqu'on utilise l'étiquette "minorité » en norvégien, la plupart des gens raisonnent en termes de quantité, mais je ne pense pas que cela doive nécessairement être corrélé à la quantité.

E.A et A.K.A. : Nous ne sommes pas d'accord. À notre avis, la minorité désigne un groupe moins nombreux que la majorité. Mais les minorités peuvent être puissantes, comme le montrent par exemple les puissants « amis " en Italie.

S.T. : Je pense que $\varnothing$. et G. ont fait une observation très importante lorsqu'ils ont montré que l'on pouvait être simultanément membre d'une majorité et d'une minorité, selon les éléments que l'on prend en considération. Peut-être 
faudra-t-il étudier cela de plus près. Le fait que les minorités jouissent de certains avantages précisément parce que ce sont des minorités serait également un sujet à creuser plus avant, en cherchant des exemples.

A.S. : Au Portugal, ce mot est également utilisé en politique pour définir un groupe qui n'est pas pris au sérieux à cause de l'aspect quantitatif. Cependant, ce qu'ils oublient, c'est que nous ne devrions pas sous-estimer le pouvoir des minorités ni ce qu'elles sont capables de faire lorsqu'elles se battent pour quelque chose. (...) Au Portugal, le cas du mirandais ${ }^{3}$ en est une bonne démonstration, tout comme le plus petit parti du Parlement, le PEV (Partido Ecologista os Verdes ${ }^{4}$ ), offre une vision plus politique du terme.

L'étudiant japonais montre que les langues sont les miroirs de l'expérience culturelle. Comme le dit Reinhart Koselleck (1972), ce sont des réservoirs de sens accumulés au cours de l'histoire. En d'autres termes, il peut manquer certaines strates de sens :

S.Y. : (...) En japonais, le mot «minorité » est toujours utilisé en référence à des nombres, c'est-à-dire qu'il se réfère toujours au quantitatif. En fait, nous l'écrivons à l'aide de kanji (caractères chinois) qui signifient " une quantité moindre » ou « un nombre plus petit». Depuis le dernier cours, je me suis demandé si nous utilisions le mot "minorité » sans faire référence à des nombres, mais jusqu'ici je n'ai pu trouver aucun exemple. En anglais, par exemple, nous pouvons utiliser le mot "minorité » pour signifier "minorité ethnique », ce qui est impossible en japonais : nous devons dire quelque chose comme « ethnie mineure ». Donc le mot « minorité » n'inclut pas de sens qualitatif en soi.

Cet exemple montre à quel point les échanges en ligne ont obligé les participants à réfléchir à leur vision originale du concept ainsi qu'aux ressemblances et aux différences attachées à ces concepts, en liaison avec des contextes nationaux et culturels spécifiques. Cette contextualisation, la plupart du temps plus implicite qu'explicite, était également associée à une historicisation, c'està-dire à la prise de conscience et à la compréhension du sens des concepts au fil du temps, à l'intérieur d'un cadre de changements socioculturels et politiques.

À la fin de la phase en ligne, les organisateurs du cours ont été agréablement surpris de l'engagement des étudiants lors des échanges et de l'intensité parfois atteinte par les discussions. Cependant, ils ont dû admettre que le travail s'est borné à un échange de vues et que la dernière tâche (relier les posts originaux à des définitions existantes provenant de contextes contemporains et historiques variés) n'avait pas eu l'effet systématisant et structurant attendu. L'étape de la compréhension par l'intermédiaire d'une structure n'avait pas été franchie.

3. Le mirandais (en portugais mirandês) est une langue romane dérivant directement du latin et parlée au nord-est du Portugal. Elle est la seconde langue officielle du Portugal (NdT).

4. Parti écologique Les Verts $(N d T)$. 
Il s'agissait ensuite d'utiliser les différences apparues lors de l'échange comme matériau de compréhension d'un "ordre supérieur » durant la rencontre des étudiants en avril 2013, et leur collaboration en face-à-face.

\section{Rencontre en face-à-face}

En termes d'apprentissage interculturel, le processus, au point où il était parvenu, courait le risque que la diversité des différences, bien que ressentie et appréciée, puisse être interprétée de façon arbitraire, déconnectée des contextes socioculturel et historique et des conditions dans lesquels elles apparaissent. S'il s'était arrêté là, le processus n'aurait pas exploité tout le potentiel critique contenu dans son approche méthodologique.

Dans la planification du programme destiné aux ateliers de travail conjoints des groupes d'étudiants, deux éléments étaient donc essentiels :

- une systématisation des résultats obtenus à partir des interactions en ligne ;

- une prise de conscience des débats politiques contemporains dans lesquels les interprétations de ces concepts sont remises en question.

La première tâche que les étudiants ont dû effectuer, lors de l'atelier, a été de structurer les contributions à l'interaction en ligne, de façon à mettre en lumière les différents contextes qui avaient donné forme aux sens attribués aux concepts débattus. La tâche était formulée de manière tout à fait ouverte, sans déterminer ce à quoi devrait ressembler la structure, quel contexte était considéré comme pertinent ni la façon dont les étudiants devraient parvenir à un accord sur ces questions. En d'autres termes, il s'agissait pour les étudiants de relever le défi de négocier et de coopérer de façon spontanée avec d'autres étudiants, qu'ils n'avaient rencontrés qu'en ligne.

Ce qui s'est ensuivi a été qualifié de "véritable défi " et de "difficile » par de nombreux étudiants. Les perspectives divergentes et les opinions différentes concernant les concepts ont posé problème, tout comme les différentes «cultures» de travail. Les étudiants norvégiens et luxembourgeois n'avaient aucune expérience commune de la structuration d'un processus en cours, ni de la façon de se mettre d'accord sur l'interprétation des tâches, d'établir les priorités ou de définir les procédures. Les quatre groupes de travail créés (un pour chaque concept) sont parvenus à divers degrés à mener leur tâche à bien, et leur évaluation finale a fait apparaître une ambivalence : l'impression d'avoir été confronté à "des ambitions et des méthodes de travail opposées parmi les membres du groupe "d'une part, et celle d'avoir fait l'expérience du «meilleur de la diversité » d'autre part. On peut dire de façon certaine que, par là-même, la rencontre en face-à-face a largement contribué à l'expérience d'apprentissage interculturel, tout spécialement pour les étudiants norvégiens, habitués à un environnement de travail ou d'études relativement monoculturel. 
Pour clore cette description de l'étude de cas, nous souhaitons mettre en lumière un dernier élément du processus d'apprentissage conceptuel : l'accent mis sur l'effet démultiplicateur de la méthodologie, qui se concentre sur le développement des aptitudes liées à la pensée critique et à la réflexivité, par l'intermédiaire de la contextualisation et de l'historicisation. On a ainsi demandé aux étudiants de fournir des exemples de contextes contemporains dans lesquels le concept qu'ils avaient étudié a récemment été mis en question, ou l'est encore. Les concepts de normalité, de minorité, de peuple et de communauté font-ils à l'heure actuelle partie intégrante des enjeux politiques? Comment sont-ils définis, interprétés et utilisés, et par qui ?

L'un des exemples récents du débat sur le concept de normalité a été le procès du terroriste norvégien Anders Behring Breivik. Le 22 juillet 2011, Breivik a fait exploser une voiture piégée à proximité des bâtiments gouvernementaux à Oslo puis a tué près de soixante-dix jeunes qui assistaient à un camp de l'Organisation des jeunes sociaux-démocrates sur l'île d'Utøya. Bien qu'il ait justifié ses actes par des raisons politiques, des experts psychiatres ont mis en doute ses capacités mentales. D’autres, qui réfutent cet argument, ont répondu que considérer Breivik comme un psychopathe aurait permis à la société norvégienne de s'exonérer du fait qu'il avait été créé par cette même société. On peut avancer que l'extrémisme de droite n'est pas un diagnostic médical, qui permettrait d'exclure de semblables opinions de la "normalité ».

Durant ces discussions, et à la lumière d'exemples provenant d'une grande variété de pays (dont la plupart sont européens), l'interconnexion profonde des quatre concepts dans les luttes contemporaines pour l'identité, fût-elle collective, à l'intérieur comme à l'extérieur du cadre de la nation, devient évidente.

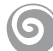

Nous avons développé les arguments en faveur de la nécessité de mettre au point des approches et des méthodologies éducatives innovantes, afin de favoriser les compétences interculturelles chez les futurs professeurs norvégiens et, ainsi, de les préparer à leur rôle professionnel d'enseignants dans différentes classes. À cet égard, le PGI a apporté la preuve de son efficacité en tant qu'outil, donnant aux apprenants l'occasion de développer leurs connaissances, leurs compétences et leurs attitudes, qui constituent toutes des composants pertinents de la compétence interculturelle.

L'historicité, l'ambiguïté et la complexité des concepts clés peut être une base fructueuse de sujets de discussion en sciences sociales en général, et plus spécifiquement en éducation à la citoyenneté et aux droits de l'homme. La méthodologie présentée dans cet article a donné de très bons résultats en matière d'apprentissage, que ce soit par la prise de conscience des sens multiples des 
concepts étudiés, mais également par l'ouverture indispensable pour accepter les ambiguïtés sans pour autant renoncer à tout jugement critique. Enfin, la composante de l'apprentissage numérique a remis en question les modes familiers de communication et donné l'occasion de s'ouvrir vers de nouvelles rencontres entre pairs. Elle a également mis en lumière ses propres limites dans ce contexte précis. Les étudiants eux-mêmes ont perçu le projet de façon très positive, déclarant en particulier qu'il les avait préparés de multiples façons à leur future pratique professionnelle.

\section{BIBLIOGRAPHIE}

BLOOM B. S. (1956) : Taxonomy of Educational Objectives, Handbook I : The Cognitive Domain. New York: David McKay Co Inc.

KOSELLECK R. (1972) : Einleitung, in : Reinhart Koselleck, Werner Conze, Otto Brunner (Hrsg.), Geschichtliche Grundbegriffe. Historisches Lexikon zur politischsozialen Sprache in Deutschland. Bd. 1, Klett-Cotta, Stuttgart 1972, p. XIII-XXVII.

KOSELLECK R. (2002): The practice of conceptual history. Timing history, spacing concepts, Stanford : Stanford University Press.

The Norwegian Directorate for Education and Training: National Curriculum for Knowledge Promotion in Primary and Secondary Education and Training/ Quality Framework, p. 3 ; http://www.udir.no/Upload/larerplaner/Fastsatte_lareplaner_for_ Kunnskapsloeftet/5/prinsipper_lk06_Eng.pdf ?epslanguage $=$ no 
